Revista Brasileira de

Engenharia Agrícola e Ambiental

v.15, n.2, p.131-138, 2011

Campina Grande, PB, UAEA/UFCG - http://www.agriambi.com.br

agriambi

Protocolo 161.09 - 22/10/2009 • Aprovado em 08/12/2010

\title{
Análise da pluviometria e dias chuvosos na região Nordeste do Brasil
}

\author{
Vicente P. R. da Silva ${ }^{1}$, Emerson R. R. Pereira ${ }^{1}$, Pedro V. de Azevedo ${ }^{1}$, \\ Francisco de A. S. de Sousa ${ }^{1} \&$ Inajá F. de Sousa ${ }^{2}$
}

\begin{abstract}
RESUMO
Os dados diários de chuva de 600 estações meteorológicas foram analisados para avaliar a variabilidade da pluviometria na região Nordeste do Brasil. No estudo foram construídos mapas do número de dias chuvosos, do coeficiente de variação (CV) da precipitação pluvial e do número de dias chuvosos. A análise de correlograma também foi aplicada aos totais anuais da precipitação pluvial na região. O s resultados evidenciam que as áreas do semiárido da região de estudo têm os maiores valores de CV da precipitação pluvial e do número de dias chuvosos que as áreas localizadas no litoral e agreste. O s maiores valores de coeficiente de variação estão associados aos menores valores de precipitação pluvial e ao número de dias chuvosos. A variabilidade da precipitação pluvial é menor nas regiôes em que os totais pluviométricos são maiores que na região do Polígono das Secas. As altas variabilidades da precipitação pluvial e do número de dias de chuva são fatores limitantes na agricultura de sequeiro nas microrregiões localizadas nas áreas semiáridas do N ordeste do Brasil. Os correlogramas de precipitação pluvial indicaram tendências significativas para o limite de confiança de $95 \%$ em algumas estações.
\end{abstract}

Palavras-chave: autocorrelograma, coeficiente de variação, semiárido

\section{An analysis of rainfall and rainy days in the northeastern region of Brazil}

\begin{abstract}
Rainfall daily data of 600 meteorological stations were analyzed to assess the changes in precipitation in northeastern Brazil. M aps of rainy day numbers and the coefficient of variation (CV) for rainfall and rainy days are presented. Autocorrelation function was obtained for the annual rainfall in northeastern Brazil. Results showed that the semiarid regions have a high coefficient of variation for both rainfall and rainy days than those located in northeastern coast and 'agreste' of Brazil. The highest values of coefficient of variation are associated to the lowest values of rainfall and rainy days. The rainfall change in northeastern Brazil is smaller in rainy regions than in dry regions. The high changes in rainfall and rainy days are limiting features to the rainfed agriculture in semiarid environments. The correlograms of rainfall indicated significant trend for the limits of $95 \%$ confidence levels on some stations.
\end{abstract}

Key words: correlograms, coefficient of variation, semi arid

UACA/UFCG, Av. Aprígio Veloso, 882 Bodocongó, CEP 58429-140, Campina Grande, PB. Fone: (83) 2101-1202; 2101-1201. E-mail: vicente@dca.ufcg.edu.br; emerson ufcg@yahoo.com.br; pvieira@dca.ufcg.edu.br; fassis@dca.ufcg.edu.br

D EA/U FS, Cidade Universitária Prof. José Aloísio de Campos, CEP 49100-000, São Cristóvão, SE. Fone: (79) 2105-6929. E-mail: ifsousa@ufs.br 


\section{INTRODUÇÃO}

A precipitação pluvial tem sido bastante estudada em diferentes regiões do mundo, em face de sua importância no ciclo hidrológico e a manutenção dos seres vivos no planeta. As secas constituem sério problema para a sociedade humana e para os ecossistemas naturais (Dinpashoh et al., 2004). Neste sentido, diferentes metodologias têm sido utilizadas para se analisar a variabilidade da precipitação pluvial. Silva et al. (2005), estudaram a variabilidade da precipitação pluvial no Estado da Paraíba, com base na teoria da entropia. Analisando a variabilidade climática no Nordeste do Brasil (NEB), com base no teste de Mann-Kendall, Silva (2004) observou tendências significativamente decrescentes em várias localidades desta região, e sugeriu que tal variabilidade pode estar relacionada com mudanças climáticas no Nordeste do Brasil, que atinge não apenas o semiárido da região mas, também, a área litorânea.

Como a variação sazonal da precipitação pluvial exerce forte influência sobre as condições ambientais, muitos pesquisadores vêm desenvolvendo estudos com base no número de dias chuvosos (Brunettia et al., 2001; Seleshi \& Zanke, 2004; Zanetti et al., 2006; Modarres \& Silva, 2007). Ainda sobre este assunto, Hess et al. (1995) registraram que o decréscimo da precipitação no nordeste da zona árida da Nigéria resultou em decréscimo no número de dias chuvosos. Brunettia et al. (2001) notaram que o decréscimo no número de dias chuvosos na Itália é mais significante no estudo da intensidade de precipitação do que os totais anuais. As regiões semiáridas têm, como característica principal, as chuvas irregulares, variando espacialmente e de um ano para outro (Balme et al., 2006). O Nordeste do Brasil é conhecido como uma região seca, em que a maioria da população sobrevive da agricultura de sequeiro. O sucesso das culturas implantadas depende da regularidade e quantidade das chuvas. As variabilidades espacial e temporal da precipitação pluvial nas regiões áridas e semiárido são fatores limitantes para a agricultura de sequeiro (Graef \& Haigis, 2001). A precipitação pluvial pode variar consideravelmente, até mesmo dentro de alguns quilômetros de distância e em escalas de tempo diferentes, tornando as colheitas das culturas imprevisíveis. A maior parte da região Nordeste do Brasil se situa dentro da zona semiárida, com grandes problemas para a sociedade e para os ecossistemas naturais, decorrentes das secas periódicas.

O sucesso da produção agrícola de determinadas culturas, sobretudo em áreas que não são irrigadas, depende muito do regime pluviométrico local. Nas áreas em que a distribuição de chuva no tempo e no espaço não é regular, a cultura pode sofrer déficit hídrico. Visando estabelecer um estudo nos regimes pluviométricos da região Nordeste do Brasil e viabilizar um controle melhor no monitoramento dos recursos hídricos, várias metodologias têm sido utilizadas para se analisar a variabilidade da precipitação pluvial (Balme et al., 2006). As regiões semiáridas têm, como característica principal, as chuvas irregulares, variando espacialmente e de um ano para outro, não obstante a existência de modelos hidrológicos para estimar a precipitação pluvial. Entretanto, a sensibilidade dos modelos hidrológicos distribuídos em diferentes padrões, responde apenas pela distribuição espacial da precipitação pluvial (Arnaud et al., 2002).
O conhecimento do regime de precipitação pluvial de uma região, no que concerne à duração e ao final da estação chuvosa, e a possibilidade de se conhecer períodos mais susceptíveis a estiagem (veranicos) dentro dessa estação, são fundamentais para a elaboração de um calendário agrícola e a implementação de projetos agrícolas. No contexto da agricultura de sequeiro, Azevedo \& Silva (2007) destacam que a estação de crescimento das culturas depende da época em que as chuvas efetivamente começam. Os períodos chuvosos com início precoce são, em geral, potencialmente superiores na produtividade das culturas em relação aos períodos chuvosos de início tardio. Machado et al. (1996) também atentaram para a importância do comportamento irregular do período chuvoso para a agricultura quando estudaram a duração da estação chuvosa no Estado de Minas Gerais. A parte semiárida do NEB apresenta vários aspectos positivos para o cultivo de diversas culturas, devido principalmente à disponibilidade energética, muito embora ofereça restrições relacionadas às disponibilidades hídricas (Silva et al., 2007). As variações extremas em eventos de precipitação pluvial influenciam na vida humana e nas atividades agrícolas em diferentes regiões do planeta. Assim, é necessário conhecer a distribuição e a variabilidade da precipitação pluvial em variadas épocas do ano. Com base no Número de Dias de Chuva (NDC) é possível se obter uma idéia da intensidade da precipitação pluvial, haja vista que a análise do total de chuva em intervalos de tempo distintos revela sua intensidade e a variabilidade quantitativa e qualitativa (Barnston \& Smith 1996). Segundo Fischer et al. (2008) com o NDC é possível se ter uma idéia da intensidade da precipitação pluvial pois, ao se analisar o total de chuvas em intervalos de tempo distintos, é possível se obter a intensidade e a variabilidade quantitativa desta variável.

As irregularidades no regime pluviométrico são provocadas pelas mudanças da frequência e/ou intensidade dos eventos de precipitação. O melhor entendimento do comportamento da precipitação pluvial, com vistas ao seu aproveitamento máximo nas atividades agrícolas, pode ser obtido com o estudo do número de dias de chuva. A variabilidade espacial e temporal da precipitação pluvial no Nordeste do Brasil, apesar dos estudos aqui mencionados, ainda tem sido pouco estudada apesar de sua importância para o propósito de formulação de estratégias de combate aos efeitos da seca no semiárido. Neste contexto, o presente trabalho tem como objetivo: analisar a variabilidade espacial e temporal da precipitação pluvial e do número de dias de chuva no Nordeste do Brasil e avaliar a aleatoriedade das séries temporais de precipitação pluvial e do número de dias de chuva.

\section{Material e MÉTOdos}

\section{Localização da área de estudo}

A região Nordeste do Brasil (NEB) está situada entre os paralelos de $01^{\circ} 02^{\prime} 30^{\prime \prime}$ de latitude norte e $18^{\circ} 20^{\prime} 07^{\prime}$ ' de latitude sul e entre os meridianos de $34^{\circ} 47^{\prime} 30^{\prime \prime}$ e $48^{\circ} 45^{\prime} 24^{\prime \prime}$ a oeste do meridiano de Greenwich. Esta região se limita ao norte e ao leste, com o Oceano Atlântico; ao sul, com os Estados de Minas Gerais e Espírito Santo e a oeste com os Estados do Pará, 
Tocantins e Goiás (Silva et al., 2006). Esta região tem 1.558.196 $\mathrm{km}^{2}$, equivalentes a $18 \%$ do território nacional e possui a maior costa litorânea do País. Dentre os estados nordestinos a Bahia tem a maior costa litorânea, com 932 km e o Estado do Piauí apenas $60 \mathrm{~km}$ de litoral.

O NEB é dividido em quatro zonas geográficas: (i) MeioNorte: é a faixa de transição entre a Amazônia e o sertão, abrange os Estados do Maranhão e Piauí; é chamada, também, de Mata dos Cocais, em virtude das palmeiras de babaçu e carnaúba. No litoral chove cerca de $2.000 \mathrm{~mm}$ anuais, sendo que mais para o leste e/ou para o interior este número cai para $1.500 \mathrm{~mm}$ anuais; já no sul do Piauí, uma região mais parecida com o sertão, só chove em média $700 \mathrm{~mm}$ por ano; (ii) Sertão: fica localizado no interior do Nordeste e possui clima semiárido. Esta mesorregião alcança o norte de Minas Gerais, no Sudeste do NEB, com chuvas irregulares e escassas, além de períodos de estiagem, e a vegetação típica é a caatinga; (iii) Agreste Nordestino: é uma zona de transição entre a Zona da Mata e o Sertão, localizado no alto do planalto da Borborema, que é um obstáculo natural para a chegada das chuvas até o sertão pois ela se estende do sul da Bahia até o Rio Grande do Norte. O principal acidente geográfico da região é o planalto da Borborema. Do lado leste do planalto estão as terras mais úmidas (Zona-da- Mata) enquanto para o interior o clima vai ficando cada vez mais seco (sertão); (IV) Zona da Mata: localizada no leste, entre o planalto da Borborema e a costa litorânea, e se estende desde o Rio Grande do Norte até o sul da Bahia, onde as chuvas são mais abundantes. Nesta área os cultivos de cana-de-açúcar e cacau substituiram as áreas de floresta (IBGE, 2000).

Três dos quatro tipos de clima que existem no Brasil estão presentes na região Nordeste: (i) Clima Equatorial Úmido: presente em uma pequena parte do Estado do Maranhão e na divisa com o Estado do Pará; (ii) Clima Litorâneo Úmido: presente do litoral do Estado da Bahia ao Rio Grande do Norte; (iii) Clima Tropical: presente nos Estados da Bahia, Ceará, Maranhão e Piauí e (iv) Clima Tropical Semiárido: presente em todo o sertão nordestino (Silva et al., 2002).

\section{Análise estatística}

Neste estudo foram utilizadas 600 séries temporais diárias de precipitação pluvial dos nove estados da região Nordeste do Brasil, com mais de 30 anos de dados, contínuos e sem falhas. Considerou-se dia chuvoso aquele com precipitação pluvial acima de $0,1 \mathrm{~mm}$. Mapas do número de dias chuvosos e dos coeficientes de variação da precipitação pluvial e do número de dias chuvosos foram construídos, utilizando-se o software SURFER, versão 7.0, com base no método de interpolação "Kriging". Na identificação dos períodos seco e chuvoso foram construídos histogramas de frequência da precipitação pluvial para cada posto pluviométrico.

\section{Coeficiente de variação}

O coeficiente de variação $(\mathrm{CV})$ para cada série de dados do posto pluviométrico analisado foi calculado pela relação entre o desvio padrão e a média aritmética. O CV (\%) da série de dados é uma medida de dispersão da variável analisada, obtido pela equação (Jensen \& Pedersen, 2005):

$$
\mathrm{CV}=\frac{\sigma}{\mu} \times 100=\frac{\left[\frac{1}{n-1}\left[\sum_{i=1}^{n} x_{i}^{2}-\frac{1}{n}\left(\sum_{i=n}^{n} x_{i}\right)^{2}\right]\right]^{1 / 2}}{\frac{1}{n} \sum_{i=n}^{n} x_{i}} \times 100
$$

em que ó é o desvio padrão e $\mu$ é a média da série temporal da precipitação pluvial, $\mathrm{n}$ é o tamanho da série temporal e Xi é o valor da precipitação pluvial.

\section{Função de autocorrelação}

Os métodos de análise de séries temporais no domínio temporal procuram caracterizar as séries de dados nos mesmos termos em que são observadas e registradas. A ferramenta primária para a caracterização de relações entre valores de dados na aproximação do domínio temporal é a função de autocorrelação ou coeficiente de correlação serial. A autocorrelação pode ser definida como o grau de variação comum entre uma variável $(\mathrm{X})$ medida no tempot com ela mesma medida em tempo posterior $\mathrm{t}+\mathrm{k}$, onde $\mathrm{k}$ é o tempo do intervalo de observação. A autocorrelação mede a persistência de uma onda dentro de uma série temporal. Quando a autocorrelação é próxima de zero ela informa que há um processo randômico ocorrendo sem nenhuma persistência ou regularidade. A autocorrelaçãoé calculada, normalmente, para um lag (intervalo) de variação e o resultado é plotado em um gráfico que inclui os valores obtidos versus $k$ intervalos de observação da variável. Para o caso especial do lag zero, que corresponde ao cálculo da autocorrelação do primeiro dado da série com ele mesmo, o resultado é 1 . O gráfico da função autocorrelação versus "lag" é chamado correlograma. O correlograma é útil para determinar se sucessivas observações são independentes. Se o correlograma indicar alta correlação entre $\mathrm{X}(\mathrm{t}) \mathrm{e} \mathrm{X}(\mathrm{t}+\hat{\mathrm{o}})$, as observações não podem ser assumidas como independentes. Desta forma, a função de autocorrelação pode indicar a memória de um processo estocástico. A aleatoriedade dos dados das séries temporais foi avaliada com base na função de autocorrelação para os intervalos de confiança (IC) dada por (Modarres \& Silva, 2007):

$$
\mathrm{IC}=\frac{\mathrm{Z}_{\mathrm{i}}-\alpha / 2}{\sqrt{\mathrm{n}}}
$$

em que $Z$ é a função da distribuição normal, n é o tamanho da amostra e a é o nível de significação. O intervalo de confiança depende do tamanho de amostra. O teste de Mann-Kendall foi aplicado para avaliar as tendências das séries temporais, que foram removidas quando detectadas. A normalidade das séries temporais foi avaliada, também, antes da elaboração dos correlogramas. Os procedimentos para a utilização do teste de Mann-Kendall podem ser encontrados em Santos et al. (2010). 


\section{RESULTADOS E DISCUSSÃO}

Os mapas de isolinhas do número de dias de chuva e dos coeficientes de variação $(\mathrm{CVs})$ da precipitação pluvial e do número de dias de chuvas na região Nordeste do Brasil são apresentados na Figura 1. Nas localidades em que a precipitação pluvial é baixa, ocorreram altos valores de CVs e, para as localidades em que a precipitação pluvial é bastante alta, os valores de CVs

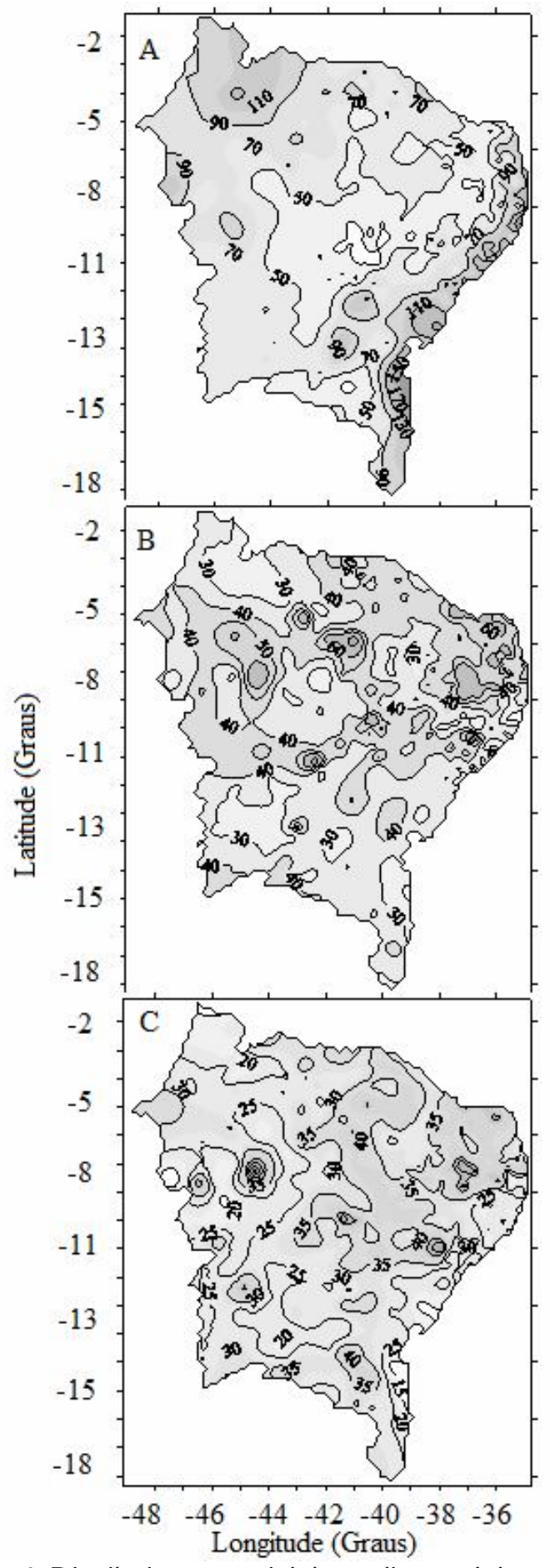

Figura 1. Distribuição espacial da média anual do número de dias de chuva (A), do coeficiente de variação da precipitação pluvial anual (B) e do coeficiente de variação do número de dias de chuva (C) no N ordeste do Brasil foram baixos. Resultados semelhantes obtiveram Dinpashoh et al. (2004) em estudo realizado para o Irã, em que os valores da média da precipitação pluvial são inversamente proporcionais aos valores dos CVs. A análise dos resultados revela, ainda, que a distribuição da precipitação pluvial e do número de dias chuvosos para o Nordeste do Brasil não é uniforme e varia sensivelmente de acordo com a localização geográfica.

No período estudado o maior número de dias com chuvas se concentra na costa leste e agreste, com valores entre 90 e 170 dias; em seguida, decresce em direção ao meio norte do Nordeste do Brasil, com valores entre 70 e 120 dias. Na parte do semiárido nordestino foram encontrados os menores valores do número de dias de chuva, atingindo o máximo de 90 dias (Figura 1A). Os valores de CVs são muito baixos na zona da mata e no agreste nordestino para as duas variáveis analisadas, oscilando entre 20 e $60 \%$ para precipitação pluvial (Figura 1B) e entre 15 e $45 \%$ para o número de dias de chuva (Figura 1C). No meio norte do Nordeste do Brasil os valores de CVs variaram entre 30 e $60 \%$ para precipitação pluvial e entre 20 e $45 \%$ para o número de dias de chuva. Na parte do semiárido nordestino os valores de CVs variaram entre 35 e $90 \%$ para precipitação pluvial e entre 25 e $80 \%$ para o número de dias de chuva. Os altos valores dos CVs de precipitação pluvial e do número de dias de chuvas na parte semiárida são atribuídos aos baixos índices pluviométricos nesta extensa área do Nordeste. Valores semelhantes de CVs foram encontrados no Irã, por Dinpashoh et al. (2004), com coeficientes de variação da precipitação pluvial variando entre $18 \%$ ao Norte, onde se situam as regiões montanhosas, e $75 \%$ no Sul do país.

Os valores médios do número de dias chuvosos e dos coeficientes de variação da precipitação pluvial e do número de dias chuvosos (NDC) das estações meteorológicas dos nove estados do Nordeste do Brasil analisados neste estudo são apresentados nas Tabelas 1, 2 e 3, respectivamente. Os maiores valores médios de NDC para o Nordeste do Brasil se concentram, em sua maior parte, no período de janeiro e junho (Tabela 1). Para os Estados do Maranhão e Piauí observa-se que a média do NDC variou entre 1 e 18 dias. A estação chuvosa em todos os estados do NEB culmina com a época do ano em que a Zona de Convergência Intertropical (ZCIT) está mais ao Sul, que corresponde aos meses de março, abril e maio (trimestre chuvoso), voltando a se constatar um crescimento do NDC nos meses de outubro, novembro e dezembro. Este crescimento está associado à formação de ZCAS (Zona de Convergência do Atlântico Sul) (Uvo \& Nobre, 1989). Para os Estados do Ceará e Rio Grande do Norte, os valores médios de NDC variaram entre 1 e 14 dias. O Estado do Ceará e a microrregião do Sertão do Rio Grande do Norte estão inseridos no semiárido nordestino, onde a estação chuvosa, em sua maior parte, culmina com a época do ano em que a ZCIT está mais ao Sul, que corresponde aos meses de março, abril e maio (trimestre chuvoso). Para os Estados da Paraíba e Pernambuco, a média de NDC variou de 2 a 9 dias. Para esses dois estados do NEB a precipitação pluvial e o número de dias de chuva são intensificados por dois fenômenos atmosféricos: pela ZCIT, no semiárido, e pelos Distúrbios Ondulatórios de Leste (DOL), na microrregião do litoral (Silva et al., 2005). Para os Estados de Alagoas e Sergipe a média de NDC variou entre 3 e 16 dias de 
Tabela 1. Média do número de dias chuvosos em cada estado da região Nordeste do Brasil para os meses do ano e períodos anual, chuvoso e seco

\begin{tabular}{lrrrrrrrrrrrrrrr}
\hline Estado & Jan & Fev & Mar & Abr & Mai & J un & Jul & Ago & Set & Out & Nov & Dez & Anual & Chuvoso & Seco \\
Maranhão & 14 & 15 & 18 & 16 & 10 & 5 & 3 & 2 & 2 & 4 & 5 & 8 & 101 & 14 & 3 \\
Piaú́ & 9 & 11 & 12 & 10 & 4 & 2 & 1 & 1 & 1 & 3 & 5 & 6 & 73 & 9 & 1 \\
Ceará & 7 & 10 & 14 & 13 & 8 & 4 & 2 & 1 & 1 & 1 & 1 & 3 & 66 & 10 & 1 \\
R.G.do Norte & 5 & 7 & 11 & 11 & 8 & 6 & 5 & 5 & 5 & 5 & 4 & 4 & 63 & 8 & 1 \\
Paraíba & 4 & 6 & 9 & 9 & 8 & 7 & 6 & 4 & 3 & 2 & 2 & 3 & 63 & 7 & 2 \\
Pernambuco & 5 & 6 & 8 & 7 & 7 & 7 & 7 & 4 & 3 & 2 & 3 & 4 & 62 & 8 & 2 \\
Alagoas & 5 & 5 & 8 & 11 & 14 & 15 & 16 & 12 & 8 & 5 & 3 & 4 & 109 & 14 & 5 \\
Sergipe & 4 & 6 & 8 & 11 & 15 & 15 & 16 & 13 & 8 & 5 & 4 & 4 & 109 & 14 & 6 \\
Bahia & 6 & 6 & 9 & 7 & 6 & 5 & 5 & 5 & 4 & 4 & 7 & 7 & 75 & 9 \\
\hline
\end{tabular}

Tabela 2. M édia do coeficiente de variação da precipitação pluvial em cada estado da região Nordeste do Brasil para os meses do ano e períodos anual, chuvoso e seco

\begin{tabular}{lrrrrrrrrrrrrrrr}
\hline Estado & Jan & Fev & Mar & Abr & \multicolumn{1}{c}{ Mai } & J un & Jul & Ago & Set & Out & Nov & Dez & Anual & Chuvoso & Seco \\
Maranhão & 58 & 52 & 45 & 58 & 76 & 117 & 157 & 168 & 137 & 115 & 93 & 80 & 34 & 57 & 140 \\
Piauí & 71 & 69 & 66 & 79 & 128 & 213 & 290 & 312 & 235 & 141 & 106 & 92 & 44 & 62 & 213 \\
Ceará & 84 & 70 & 52 & 61 & 80 & 106 & 138 & 224 & 245 & 239 & 209 & 135 & 40 & 66 & 192 \\
R.G.do Norte & 99 & 94 & 67 & 75 & 85 & 98 & 126 & 194 & 236 & 309 & 263 & 171 & 51 & 76 & 215 \\
Paraíba & 96 & 93 & 84 & 85 & 90 & 102 & 119 & 202 & 206 & 243 & 213 & 162 & 53 & 92 & 187 \\
Pernambuco & 94 & 90 & 74 & 84 & 102 & 108 & 129 & 183 & 183 & 168 & 123 & 113 & 38 & 77 & 159 \\
Alagoas & 97 & 96 & 93 & 75 & 68 & 62 & 65 & 73 & 86 & 133 & 131 & 135 & 41 & 62 & 115 \\
Sergipe & 98 & 98 & 90 & 73 & 66 & 54 & 55 & 57 & 80 & 107 & 128 & 115 & 35 & 57 & 101 \\
Bahia & 93 & 92 & 92 & 93 & 141 & 198 & 206 & 226 & 173 & 118 & 84 & 88 & 37 & 73 & 181 \\
\hline
\end{tabular}

Tabela 3. M édia do coeficiente de variação do número de dias com chuva em cada estado da região N ordeste do Brasil para os meses do ano e períodos anual, chuvoso e seco

\begin{tabular}{|c|c|c|c|c|c|c|c|c|c|c|c|c|c|c|c|}
\hline Estado & Jan & Fev & Mar & $A b r$ & Mai & Jun & Jul & Ago & Set & Out & Nov & Dez & Anual & Chuvoso & Seco \\
\hline Maranhão & 35 & 32 & 26 & 36 & 52 & 86 & 116 & 120 & 98 & 79 & 64 & 51 & 25 & 35 & 101 \\
\hline Piauí & 48 & 45 & 41 & 54 & 92 & 149 & 220 & 240 & 156 & 100 & 70 & 65 & 32 & 48 & 157 \\
\hline Ceará & 63 & 53 & 37 & 43 & 59 & 83 & 111 & 181 & 202 & 193 & 157 & 102 & 34 & 48 & 150 \\
\hline R.G.do Norte & 87 & 71 & 50 & 54 & 62 & 77 & 98 & 147 & 186 & 235 & 192 & 125 & 43 & 57 & 163 \\
\hline Paraíba & 82 & 76 & 57 & 60 & 65 & 74 & 86 & 121 & 145 & 171 & 145 & 111 & 43 & 68 & 135 \\
\hline Pernambuco & 64 & 67 & 54 & 61 & 75 & 83 & 97 & 144 & 134 & 118 & 90 & 79 & 33 & 56 & 118 \\
\hline Alagoas & 68 & 72 & 58 & 48 & 41 & 35 & 38 & 45 & 59 & 93 & 89 & 85 & 28 & 39 & 77 \\
\hline Sergipe & 68 & 68 & 58 & 45 & 39 & 35 & 34 & 39 & 55 & 72 & 75 & 74 & 25 & 37 & 66 \\
\hline Bahia & 69 & 66 & 65 & 67 & 110 & 167 & 161 & 195 & 135 & 84 & 60 & 62 & 31 & 57 & 152 \\
\hline
\end{tabular}

chuvas. Por outro lado, para o Estado da Bahia observa-se que a média de NDC variou entre 4 e 9 dias. O regime de chuvas no Estado da Bahia está associado à ZCAS enquanto os DOL atuam na zona costeira do estado.

$\mathrm{Na}$ Tabela 2 se constatam os valores médios dos CVs da precipitação pluvial durante os meses do ano, períodos anual, chuvoso e seco, para os nove estados do Nordeste do Brasil. Os menores valores dos CVs estão localizados, em sua maior parte, no primeiro semestre do ano, em face da estação da região se concentrar basicamente neste período. Para os Estados do Maranhão e Piauí, verificaram-se valores de CV de precipitação pluvial entre 45 e $312 \%$, já para os Estados do Ceará e Rio Grande do Norte se observam valores médios de CVs de precipitação pluvial variando de 52 a 309\%. Para os Estados da Paraíba e Pernambuco os valores de CVs de precipitação variaram em média entre 74 e $243 \%$, enquanto para Alagoas e Sergipe os CVs variaram entre 54 e 135\%. No Estado da Bahia os valores de CVs de precipitação pluvial foram entre 84 e $226 \%$ durante o ano e, para o total anual, o CV da precipitação pluvial revela valores médios de 34 a $53 \%$. No período chuvoso os valores de CVs de precipitação pluvial variaram entre 57 e $92 \%$; entretanto, para o período seco eles foram entre 101 e $215 \%$. A Tabela 3 apresenta os valores médios dos CVs do número de dias de chuvas para os nove estados do Nordeste do Brasil (mensal, períodos anual, chuvoso e seco). Os menores valores de CV estão localizados, em sua maior parte, no primeiro semestre do ano. Nos Estados do Maranhão e Piauí verificaram-se valores dos CVs de número de dias de chuva entre 26 e $240 \%$, em média. Os Estados do Ceará e Rio Grande do Norte apresentaram valores médios dos CVs do número de dias de chuva variando de 37 a $235 \%$.

Nos Estados da Paraíba e Pernambuco os valores de CVs do número de dias de chuva foram entre 54 e 171\%, em média, já nos Estados de Alagoas e Sergipe, os valores médios dos CVs do número de dias de chuva variaram entre 35 e $93 \%$. Por outro lado, no Estado da Bahia os valores de CVs do número de dias de chuva estiveram entre 60 e 195\%. Para o total anual, o $\mathrm{CV}$ do número de dias de chuva revela valores médios entre 25 e $43 \%$. No período chuvoso os valores do CVs do número de dias de chuva variaram entre 35 e $68 \%$, sendo que para o período seco esses valores variaram entre 63 e $163 \%$. A Tabela 4 exibe a precipitação pluvial média em cada estado da região Nordeste do Brasil para os meses do ano e períodos anual, chuvoso e seco. 
Tabela 4. Precipitação pluvial média (mm) em cada estado da região $\mathrm{N}$ ordeste do Brasil para os meses do ano e períodos anual, chuvoso e seco

\begin{tabular}{|c|c|c|c|c|c|c|c|c|c|c|c|c|c|c|c|}
\hline Estado & Jan & Fev & Mar & Abr & Mai & Jun & Jul & Ago & Set & Out & Nov & Dez & Anual & Chuvoso & Seco \\
\hline Maranhão & 201 & 240 & 309 & 271 & 183 & 101 & 71 & 26 & 21 & 39 & 64 & 110 & 1632 & 220 & 17 \\
\hline Piauí & 160 & 189 & 226 & 153 & 42 & 8 & 4 & 4 & 11 & 47 & 95 & 104 & 1042 & 177 & 8 \\
\hline Ceará & 93 & 169 & 276 & 237 & 135 & 52 & 20 & 6 & 6 & 8 & 16 & 38 & 1056 & 199 & 8 \\
\hline R.G.do Norte & 48 & 89 & 168 & 169 & 114 & 91 & 70 & 28 & 12 & 6 & 6 & 17 & 817 & 141 & 7 \\
\hline Paraíba & 52 & 84 & 147 & 154 & 131 & 115 & 100 & 53 & 27 & 13 & 15 & 25 & 917 & 133 & 15 \\
\hline Pernambuco & 50 & 73 & 118 & 136 & 108 & 110 & 104 & 56 & 34 & 24 & 23 & 39 & 875 & 102 & 21 \\
\hline Alagoas & 53 & 55 & 122 & 177 & 223 & 188 & 156 & 101 & 62 & 38 & 29 & 36 & 1096 & 153 & 37 \\
\hline Sergipe & 41 & 54 & 87 & 131 & 178 & 164 & 166 & 97 & 61 & 41 & 41 & 41 & 1066 & 118 & 44 \\
\hline Bahia & 91 & 97 & 137 & 125 & 99 & 83 & 82 & 55 & 46 & 58 & 103 & 107 & 978 & 115 & 41 \\
\hline
\end{tabular}

A função de autocorrelação aplicada às series temporais aleatória de precipitação pluvial para uma localidade de cada estado da região Nordeste do Brasil indicou valores significativos nas autocorrelações das séries temporais para o limite de $95 \%$ de confiança (Figura 2). O posto pluviométrico do Alto Parnaíba, no Estado do Maranhão, apresentou apenas um valor significativo de autocorrelação, o de lag 2. Esta figura revela, ainda, que do oitavo ao décimo segundo ano ocorre certa persistência da precipitação pluvial (Figura 2A).

Analisando os correlogramas para o Estado do Piauí (Figura 2B), verificou-se que o posto pluviométrico de Bom Princípio apresentou valores de autocorrelações significativos nos lags 1 e 8 , indicando persistência da precipitação pluvial no intervalo de 8 anos. Também se notou certo grau de persistência e estacionariedade das séries temporais, que são quase aleatórias.

Por outro lado, os valores de autocorreleção de Almofada, no Estado do Ceará (Figura 2C), revelam que esta série temporal é quase cíclica, estacionária e com sazonalidade de três anos, com destaque para os lags 5 e 11, enquanto os outros valores ocorrem de forma quase aleatória. Os valores de autocorrelação dos postos de Apodi, no Estado do Rio Grande do Norte, são
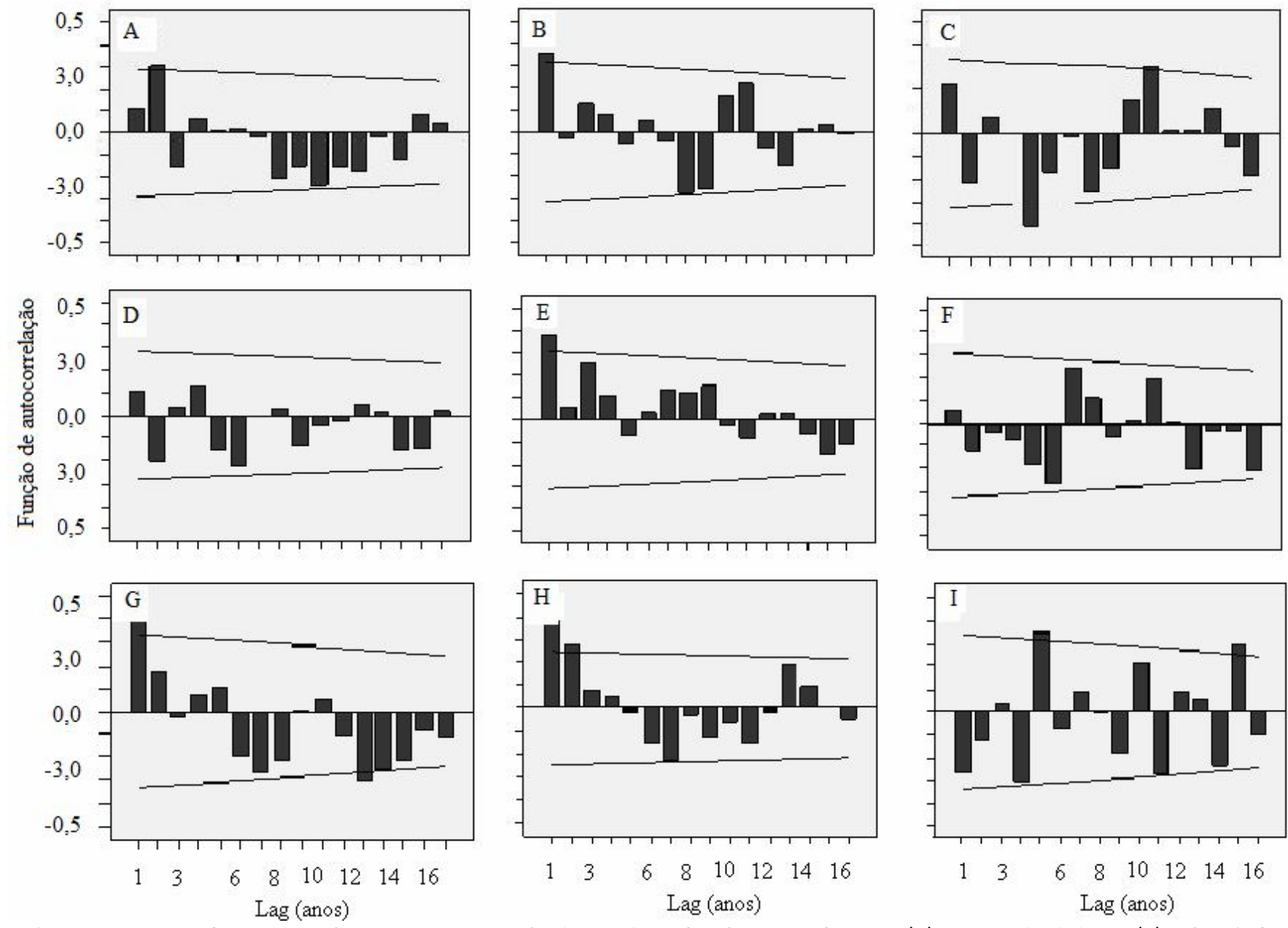

Figura 2. Função de auto correlação para postos pluviométricos do Alto Parnaíba, MA (A); Bom Principio, PI (B); Almofada, CE (C); Apodi, RN (D); Alagoa Grande, PB (E); Juazeiro dos Cândidos, PE (F); Maragogi, AL (G); Aracaju, SE (H) e Barreiras, BA (I) 
típicos de séries temporais quase aleatórias (Figura 2D). É provável que as chuvas desse posto sejam favorecidas pela brisa marítima, em face do seu regime pluviométrico ser completamente diferente das demais áreas do Estado. Os valores de autocorrelação do posto de Alagoa Grande, PB, indicam séries temporais quase aleatórias, destacando-se o valor da autocorrelação de lag 1 com significância estatística a nível de 95\% de probabilidade (Figura 2E). Os baixos valores das autocorrelações dos outros lags implicam na fraca persistência dos totais anuais dos eventos de chuva no Estado. A análise do correlograma do posto pluviométrico do Estado de Pernambuco revela que a série temporal de Juazeiro dos Cândidos é completamente aleatória no período estudado (Figura 2F).

Portanto, os eventos de precipitação pluvial nesta localidade são quase aleatórios, com períodos chuvosos similares no tempo. No posto de Maragogi, no Estado de Alagoas, os valores das autocorrelações foram significativos para os lags 1, 12 e 13 (Figura G). A distribuição dos lags ao longo do tempo indica que esta série temporal apresenta curtos períodos de correlação - três anos; já o posto de Aracaju, no Estado de Sergipe, apresentou valores de autocorrelação significativos para os primeiros lags (Figura 2H). As séries temporais desses correlogramas são estacionárias, com curtos períodos de correlação. O posto de Barreiras, no Estado da Bahia, exibe valores significativos de autocorrelação para os lags 5, 11 e 15, indicando certa dependência nas observações com intervalos aproximados de cinco anos (Figura 2I).

\section{ConclusõEs}

1. A variabilidade da precipitação pluvial não é uniforme em todo o Nordeste do Brasil. A região do semiárido nordestino tem os maiores valores de coeficiente de variação da precipitação pluvial e número de dias de chuva do que aqueles apresentados no litoral e agreste nordestino.

2. Os maiores valores do coeficiente de variação estão associados aos menores valores de precipitação pluvial e número de dias de chuva. A variabilidade da precipitação pluvial no Nordeste do Brasil é menor no período chuvoso que no período seco.

3. Os maiores valores de número de dias de chuva em grande parte da região Nordeste do Brasil ocorrem no primeiro semestre do ano, em face da atuação da ZCIT e do DOL.

4. A análise dos coeficientes de autocorrelação revela que o comportamento da precipitação pluvial no Nordeste do Brasil é quase aleatório.

\section{LITERATURA CITADA}

Arnaud, P.; Bouvier, C.; Cisneros, L.; Dominguez, R. Influence of rainfall spatial variability on flood prediction. Journal of Hydrology, v.297, p.109-123, 2002.
Azevedo, P. V. de; Silva, F. D. S. Risco climático para o cultivo do algodoeiro na região Nordeste do Brasil. Revista Brasileira de Meteorologia, v.22, p.408-416, 2007.

Balme, M.; Vischel, T.; Lebel, T.; Peugeot, C.; Galle, S. Assessing the water balance in the Sahel: Impact of small scale rainfall variability on runoff. Part 1: Rainfall variability analysis. Journal of Hydrology, v.33, p.336-348, 2006.

Barnston, A. G.; Smith, T. M. Specification and prediction of global surface temperature and precipitation from global SST using CCA. Journal of Climate, v.9, p.2660- 2697, 1996.

Brunettia, M.; Maugerib, M.; Nannia, T. Changes in total precipitation, rainy days and extreme events in northeastern Italy. International Journal of Climatology, v.21, p.861-871, 2001.

Dinpashoh, Y.; Fakheri-Fard, A.; Moghaddam, M.; Jahanbakhsh, S.; Mirnia, M. Selection of variables for the purpose of regionalization of Iran's precipitation climate using multivariate methods. Journal of Hydrology, v.297, p.109-123, 2004.

Fischer, G. R.; Diniz, G. B.; Marques, J. R. Q. Previsão do número de dias de chuva para a metade sul do Rio Grande do Sul utilizando a temperatura da superfície do mar (TSM). Revista Brasileira de Meteorologia, v.23, p.143-151, 2008.

Graef, F.; Haigis, J. Spatial and temporal rainfall variability in the sahel and it's effects on formen management strategies. Journal of Arid Environments, v.48, p.221-231, 2001.

Hess, T. M.; Stephens, W.; Maryah, U. M. Rainfall trends in the North East arid zone of Nigeria 1961-1990. Agricultural and Forest Meteorology, v.74, p.87-97, 1995.

IBGE - Instituto Brasileiro de Geografia e Estatística. Geografia do Brasil. Região Nordeste. Rio de Janeiro: SERGRAF, 2000. 466p.

Jensen, N. E.; Pedersen, L. Spatial variability of rainfall: Variations within a single radar pixel. Atmospheric Research, v.77, n.1, p.269-277, 2005.

Machado, M. A. M.; Sediyama, G. C.; Costa, J. M. N.; Costa, M. H. Duração da estação chuvosa em função das datas de início do período chuvoso para o estado de Minas Gerais. Revista Brasileira de Agrometeorologia, v.4, p.73-79, 1996.

Modarres, R.; Silva, V. de P. R. da. Rainfall trends in arid and semi-arid regions of Iran. Journal of Arid Environments, v.70, p.344-355, 2007.

Santos, D.N.; Silva, V. de P. R. da; Sousa, F. de A. S. de; Silva, R. A. Estudo de alguns cenários climáticos para o Nordeste do Brasil. Revista Brasileira de Engenharia Agrícola e Ambiental, v.14, p.492-500, 2010.

Seleshi, Y.; Zanke, U. Recent changes in rainfall and rainy days in Ethiopia. International Journal of Climatology, v.24, p.973983, 2004.

Silva, V. de P. R. da. On climate variability in Northeast of Brazil. Journal of Arid Environments, v.58, p.574-596, 2004.

Silva, V. de P. R. da; Azevedo, P. V. de; Silva, B. B. da. Surface energy and evapotranspiration of a mango orchard frown in a semiarid environment. Agronomy Journal, v.1, p.13911396, 2007.

Silva, V. de P. R. da; Belo Filho, A. F.; Silva, B. B. da; Campos, J. H. B. C. Desenvolvimento de um sistema de estimativa da evapotranspiração de referência. Revista Brasileira de Engenharia Agrícola e Ambiental, v.9, p.547-553, 2005. 
Silva, V. de P. R. da; Guedes, M. J. F.; Lima, W. F. A.; Campos, J. H. B. C. Modelo de previsão de rendimento de culturas de sequeiro, no semi-árido do Nordeste do Brasil. Revista Brasileira de Engenharia Agrícola e Ambiental, v.6, p.83-87, 2002.

Silva, V. de P. R. da; Sousa, F. de A. S.; Cavalcanti, E. P.; Souza, E. P.; Silva, B. B. da Teleconnections between sea-surface temperature anomalies and air temperature in northeast Brazil. Journal of Atmospheric and Solar-Terrestrial Physics, v.68, p.781-792, 2006.
Uvo, C. R. B.; Nobre, C. A. Zona de convergência intertropical (ZCIT) e a precipitação no Norte do Nordeste do Brasil. Boletim de Monitoramento e Análise Climática, v.4, p. 34-40, 1989.

Zanetti, S. S.; Oliveira, V. P. S.; Pruski, F. F. Validação do modelo Clima BR em relação ao número de dias chuvosos e à precipitação total diária. Engenharia Agrícola, v.26, p.96102, 2006. 